\title{
The correlation between the extent of left upper abdominal resections and perioperative outcomes in advanced stage and relapsed ovarian cancer
}

\author{
Nicolae BACALBASA ${ }^{1,2,3}$, Irina BALESCU ${ }^{1,4}$, Camelia DIACONU ${ }^{1,5}$, Simona DIMA ${ }^{3}$, \\ Laura ILIESCU ${ }^{1,6}$, Mihai DIMITRIU ${ }^{1,7}$, Mihaela VILCU ${ }^{1,2}$, Alexandru FILIPESCU ${ }^{1,8}$, \\ Ioana HALMACIU ${ }^{9}$, Dragos CRETOIU ${ }^{10,11}$, Iulian BREZEAN ${ }^{1,2}$ \\ 1"Carol Davila" University of Medicine and Pharmacy, Bucharest, Romania \\ 2"I. Cantacuzino" Clinical Hospital, Bucharest, Romania \\ ${ }^{3}$ Center of Excellence in Translational Medicine, Fundeni Clinical Institute, Bucharest, Romania \\ ${ }^{4}$ Ponderas Academic Hospital, Bucharest, Romania \\ ${ }^{5}$ Floreasca Clinical Emergency Hospital, Bucharest, Romania \\ ${ }^{6}$ Fundeni Clinical Institute, Bucharest, Romania \\ 7"St Pantelimon" Emergency Hospital, Bucharest, Romania \\ ${ }^{8}$ Elias Emergency Hospital, Bucharest, Romania \\ 9"George Emil Palade" University of Medicine, Pharmacy, Science and Technology, Tg. Mureş, Romania \\ 10"Alessandrescu-Rusescu" National Institute of Mother and Child Health, Fetal Medicine Excellence \\ Research Center, Bucharest, Romania \\ ${ }^{11}$ Department of Cell and Molecular Biology and Histology, "Carol Davila" University of Medicine and \\ Pharmacy, Bucharest, Romania
}

\begin{abstract}
The aim of this study is to analyze the feasibility and safety of left upper abdominal resections as part of debulking surgery. Case series presentation. Between January 2015 and August 2019, 32 patients were submitted to left upper abdominal resections. Left upper abdominal resections were performed as part of primary cytoreduction in 22 cases, as part of secondary cytoreduction in eight cases, and respectively as part of tertiary cytoreduction in two cases. The complexity of the resection increased from primary to secondary and tertiary cytoreduction; in the meantime, the rates of postoperative complications also increased with the attempt of cytoreduction. Therefore the postoperative morbidity rate was of $27 \%$ at the time of primary cytoreduction, $37.5 \%$ at the time of secondary cytoreduction and $50 \%$ at the time of tertiary cytoreduction. However, association of left upper abdominal resections leaded to a complete resection rate of $77 \%$ at the time of primary cytoreduction, $62.5 \%$ at the time of secondary cytoreduction and $50 \%$ at the time of tertiary
\end{abstract}


cytoreduction. Conclusions. Left upper abdominal resections might increase the rates of complete debulking in the setting of advanced stage or relapsed ovarian cancer. However the complexity of procedures and the risk of postoperative complications increase with the number of attempts of debulking.

Keywords: left upper abdominal resections, debulking surgery, advanced stage or relapsed ovarian cancer

\section{INTRODUCTION}

Ovarian cancer is characterized by a high capacity of spread via peritoneal, lymphatic or hematogenous route, leading to the apparition of disseminated lesions even from the time of the initial diagnostic (1-4). In consequence, in such cases, extended resections might be needed in order to increase the rates of complete debulking surgery in the setting of both advanced stage and relapsed ovarian cancer (5-7). When it comes to the development of distant abdominal involvement in advanced stage or recurrent ovarian cancer, it seems that the left upper abdomen is frequently affected. In this respect, a significant number of cases will necessitate performing extended resections at this level (8-11). The aim of the current paper is to investigate the feasibility and safety of left upper abdominal resections in advanced stage or recurrent ovarian cancer, and to study the correlation ship between the degree of complexity and the perioperative outcomes of these procedures.

\section{MATERIAL AND METHODS}

Patients diagnosed with uterine neuroendocrine tumors (NET) from 1985 to present were included and were classified according to the International Federation of Obstetrics and Gynecology (FIGO) classification in early stage disease (stages I-II) and advanced stages (stages IIIIV). Medline research of the English literature between 1982 and 2020 was performed, all articles with the keywords "uterine", "neuroendocrine" "cancer" and "tumor" being reviewed. Further on, all related papers included in their reference list were also reviewed. Due to the extreme rarity of this pathology, case reports and small case series were also included.

\section{CASE SERIES PRESENTATION}

After obtaining the approval of the ethical committee (no 157/2019), data of patients submitted to debulking surgery for advanced stage or relapsed ovarian cancer were retrospectively re- viewed. Between January 2015 and August 2019, 108 such cases were identified. Among these cases there were 32 patients who necessitated left upper abdominal resections in order to maximize the debulking effort. All cases were staged according to the Federation of Obstetrics and Gynecology (FIGO) 2014 system (12) while all postoperative complications were classified according to DindoClavien scale (13). Debulking surgery was considered as complete (RO) if no visible residual disease was found at the end of resection, R1 if the maximal residual volume was smaller than $0.5 \mathrm{~cm}$ and R2 if the residual volume was larger than $0.5 \mathrm{~cm}$. In all cases debulking surgery to no visible residual disease was tempted.

Among the 108 patients who were initially analyzed, there were 85 patients submitted to primary cytoreduction, 21 cases submitted to secondary cytoreduction and three cases submitted to tertiary cytoreduction. Left upper abdominal resections were performed as part of the debulking effort in 22 cases at the time of primary cytoreduction (25.8\%), in eight cases as part of secondary cytoreduction $(38.1 \%)$ and in two cases as part of tertiary cytoreduction (66.6\%).

The mean age of patients submitted to left upper abdominal resections as part of primary cytoreduction was of 51.6 years (range $=36-62$ years), all of them being initially staged as IIIC FIGO lesions. Ascites was present at the time of initial surgery in nine cases, the mean volume being of $1,800 \mathrm{ml}$ (range $=500-4,000 \mathrm{ml}$ ). As for the histopathological types, there were 17 cases diagnosed with serous ovarian adenocarcinomas, three cases with mucinous ovarian carcinomas and other two cases with clear cell ovarian carcinomas. Details of patients submitted to left upper abdominal resections as part of primary cytoreduction are presented in the Table 1.

Although debulking surgery to no residual disease was tempted in all cases, $\mathrm{R} 0$ resections were feasible in 17 cases (77\%), other five cases being submitted to R1 resections ( 3 cases) and respectively to $\mathrm{R} 2$ resection ( 2 cases); most often the site of the residual disease was represented by the mesentery of the small bowel (in 3 cases) followed by the presence of infiltrative adenopathic lesions 
TABLE 1. Data of patients submitted to left upper abdominal resections as part of primary cytoreduction

\begin{tabular}{|l|c|}
\hline \multicolumn{1}{|c|}{ Parameter } & No. of cases \\
\hline FIGO stage & 22 \\
IIIC & \\
\hline Ascites & 9 \\
- Yes & 13 \\
\hline - No & \\
\hline Histopathological subtype & 17 \\
- Serous & 3 \\
- Mucinous & 2 \\
- Clear cell & \\
\hline Degree of differentiation & 9 \\
- G1 & 6 \\
- G2 & 7 \\
- G3 & 17 \\
\hline Type of resection & 3 \\
- R0 & 2 \\
- R1 & \\
- R2 & 17 \\
\hline Left upper abdominal resection & 9 \\
- Peritoneum & 8 \\
- Diaphragm resection & 7 \\
- Splenectomy & 3 \\
- Left colectomy & \\
- Partial gastrectomy & \\
\hline
\end{tabular}

at the level of the hepatic pedicle (in 2 cases). As for the types of visceral resections which were needed in the left upper abdomen, the most commonly encountered surgical procedures were represented by peritoneal and full thickness diaphragmatic resections followed by splenectomy (in 8 cases), left colectomy (in seven cases) and partial gastrectomy in three cases. Another important issue which should be analyzed is represented by the pattern of spread at the level of the left upper abdomen; therefore, in 15 cases the predominant pattern of spread was represented by the peritoneal route (leading to the development of disseminated nodules of peritoneal carcinomatosis) followed by the lymphatic route (in 5 cases) and by the hematogenous route in other two cases. Therefore, two of the eight cases in which splenectomy was needed proved to have a hematogenous involvement of the spleen and therefore, the final histopathological stage was of FIGO stage IV disease. However, in the remaining 20 cases the final classification was similar to the one which had been established by the imagistic studies (and was represented by FIGO stage IIIC of disease). When it comes to the postoperative outcomes, the overall morbidity was of $27 \%$, the most commonly encountered complications being related to the development of pleural effusions (in three cases) followed by pneumothorax, postoperative bleeding and abdominal abscess (each in one case).
Therefore, according to Dindo - Clavien scale, there were two grade II complications, two grade III complications and other two grade IV complications (both the case that developed postoperative bleeding and the case that developed a postoperative abdominal abscess being submitted to re-operation). However, the overall mortality was null.

As for the cases submitted to left upper abdominal resections as part of secondary cytoreduction, the mean age was of 63 years (range = 45-72 years), the median disease free survival after the initial cytoreduction being of 23 months (range $=15-32$ months). Preoperative and intraoperative details are presented in Table 2.

TABLE 2. Preoperative and intraoperative characteristics of patients submitted to left upper abdominal resections as part of secondary cytoreduction

\begin{tabular}{|l|c|}
\hline \multicolumn{1}{|c|}{ Parameter } & No. of cases \\
\hline Initial FIGO stage & 5 \\
IIIC & 3 \\
IV & \\
\hline Histopathological subtype & 6 \\
$\quad$ - Serous & 2 \\
$\quad$ - Mucinous & \\
\hline Degree of differentiation & 3 \\
- G1 & 4 \\
- G2 & 1 \\
- G3 & \\
\hline Type of resection & 5 \\
- R0 & 1 \\
- R1 & 2 \\
- R2 & \\
\hline Left upper abdominal resections & 6 \\
- Peritoneum & 5 \\
- Diaphragm resection & 5 \\
- Splenectomy & 3 \\
- Left colectomy & 3 \\
- Distal pancreatectomy & 2 \\
- Partial gastrectomy & \\
\hline
\end{tabular}

As expected, the percent of patients submitted to $\mathrm{RO}$ resections was smaller when compared to cases submitted to primary cytoreduction $(62.5 \%$ versus $77 \%)$; the most commonly encountered sites presenting unresectable disease was represented by the mesentery, followed by liver pedicle and celiac trunk. As for the type of left upper abdominal involvement, the peritoneal route was predominant in 5 cases, the lymphatic route was incriminated in two cases and the hematogenous route was incriminated in one case. Two cases who necessitated the association of pancreatic resections presented splenic hylum lesions invading the pancreatic tail, while the third case presented a pancreatic tail hematogenous metastasis. Moreover, this case also associated a splenic hematogenous metastasis, a distal spleno-pancrea- 
tectomy being performed. Postoperative morbidity at the time of secondary cytoreduction was of $37.5 \%$ and consisted of two Dindo-Clavien grade III complications and one Dindo-Clavien grade IV complication; therefore re-operation was needed in one case due to postoperative bleeding after distal spleno-pancreatectomy; however the postoperative mortality was null.

As for cases in which left upper abdominal resections were needed as part of tertiary cytoreduction, two such cases were identified. Initially one case had been diagnosed with FIGO stage IIIC ovarian cancer while the second case had been diagnosed with FIGO stage IV ovarian cancer. In both cases $\mathrm{RO}$ resections had been performed at the time of primary and respectively secondary cytoreduction; however, at the time of tertiary cytoreduction $\mathrm{RO}$ resection was achieved in only one case (50\%), the second case being submitted to an R2 resection due to the massive involvement of the hepatic pedicle. At this moment left upper abdominal resections consisted of splenectomy, partial left diaphragmatic resection and left colectomy in one case and respectively splenectomy en bloc with distal pancreatectomy, left colectomy, full thickness diaphragmatic resection and partial gastrectomy in the second case; however, the second case developed a pancreatic leak which required re-operation with poor outcomes, the patient being dead in the $34^{\text {th }}$ postoperative day due to sepsis and multiple visceral failure.

As it can be observed from the data reported above, as expected, the per cent of patients who benefited from $\mathrm{R} 0$ resection reported a significant decrease from primary to secondary and tertiary cytoreduction ( $77 \%$ versus $62.5 \%$ versus $50 \%$ ) while the rates of complex resections (defined by the need of performing multiple visceral resections including distal pancreatectomy, full thickness diaphragmatic resections and splenectomy) increased from primary to tertiary cytoreduction. In consequence, once the degree of complexity increased, the rates of postoperative morbidity and even mortality proportionally increased. However performing such extended left upper abdominal resections seems to be perfect feasible and justified in order to maximize the debulking effort, a single case being dead due to surgical complications.

\section{DISCUSSION}

The effectiveness and safety of incorporating upper abdominal resections as part of debulking surgery for advanced stage of recurrent ovarian cancer have been widely studied so far (14-18). Although initially it has been considered that the development of upper abdominal involvement is the sign of a more biologically aggressive disease, this hypothesis was rapidly destroyed by the studies which came to demonstrate that patients presenting upper abdominal involvement and who benefit from complete cytoreduction exhibit similar chances of long term survival when compared to cases presenting pelvic limited disease and significantly better outcomes when compared to cases in which although the upper abdominal involvement was diagnosed debulking effort did not comprised the lesions from the upper abdomen (15). Therefore increasing number of studies included patients submitted to different types of upper abdominal resections and demonstrated that although the rates of perioperative complications might report a certain increase, this is not a statistically significant one while the chances for long term survival are significantly improved $(1,2,5-8)$.

However, an important number of patients diagnosed with upper abdominal involvement present tumoral invasion of unresectable structures, and therefore debulking effort to no residual disease is not feasible. Therefore, most often these sites are represented by the hepatic pedicle and celiac axis as well as the diffuse involvement of the mesentery of the small bowel (19). In such cases continuing the debulking effort although complete resection is not feasible is doubtable, different opinions being reported so far $(11,20)$.

One of the most commonly encountered area of involvement in the upper abdomen is represented by the left upper abdominal quadrant, the tumoral spread occurring at this level via peritoneal, hematogenous or lymphatic route $(3,21)$. In such cases malignant involvement might induce the necessity of performing extended resections including splenectomy, pancreatic tail resection, left coloectomy, peritonectomy, full thickness diaphragmatic resections and even partial gastrectomy.

One of the first studies which were conducted on the theme of left upper abdominal resections as part of debulking surgery for advanced stage or relapsed ovarian cancer was published by Hoffman et al. in 2007 (3). The study included six patients submitted to surgery between 2002 and 2004 at the Division of Gynecologic Oncology University of South Florida, United States of America. In all cases en bloc resection of the omentum, gastro-colic ligament, colon and spleen was needed in order to achieve maximal debulking effort while in other two cases distal pancreatectomy and partial gas- 
trectomy were also associated. Postoperatively a single patient required re-operation while disease free interval ranged between 2 and 12 months. These preliminary data enabled the authors to conclude that in selected cases en bloc resections of the left upper abdominal tumoral involvement might improve the long term outcomes with acceptable rates of postoperative complications, especially if complete debulking is feasible (3).

Once the gestures of left upper abdominal resections were widely implemented as part of debulking surgery for advanced stage and relapsed ovarian cancer attention was focused on determining which the most important prognostic factors are for both short term and long term outcomes of these patients. Therefore, in a study conducted by Panici et al. the authors included 121 patients submitted to cytoreductive surgery with curative intent and demonstrated that increasing the complexity of the surgical procedure by adding pancreatic and diaphragmatic resections increased the length of hospital in stay while associating complex procedures such as pancreatectomy, diaphragmatic resections and hepatic and biliary surgical procedures were independent predictors for severe complications (11). Another study which was conducted on the theme of postoperative complications after extended upper abdominal resections for advanced stage ovarian cancer was conducted by $\mathrm{Chi}$ et al. and included 141 patients; among these patients severe complications (defined as Dindo-Clavien grade 3-5 complications) occurred in $22 \%$ of cases and were mainly related to pancreatic and diaphragmatic surgery (20).

Similarly to Panici's and Chi's studies, our data come to demonstrate that association of pancreatic resections was significantly correlated with the rates of postoperative severe complications and even death. In the meantime a significant proportion of patients included in our study developed diaphragmatic related complications (such as pleural effusions or pneumothorax) suggesting the fact that association of diaphragmatic procedures can also increase the risk of perioperative complications. However, the severity of these complications is significantly lower when compared to pancreatic surgery, conservative management being most often sufficient in order to manage them.

The fact that diaphragmatic surgery represents a crucial step as part of the cytoreductive effort has been also demonstrated in the study conducted by Fanfani et al. (22); therefore the authors underlined the fact that full thickness diaphragmatic resections were needed in $41.7 \%$ of cases submitted to primary cytoreduction, $21.6 \%$ of cases submitted to interval debulking surgery and $52.5 \%$ of cases submitted to secondary cytoreduction. Similarly to our study the necessity of performing full thickness diaphragmatic resections increased from primary to secondary cytoreduction while the most commonly encountered postoperative complication was related to the apparition of pleural effusion which was significantly associated with the need of liver mobilization and with the dimensions of the diaphragmatic disease (22).

As for the association of pancreatic surgery, it seems that this surgical procedure is an effective one in order to maximize the debulking effort in the setting of both advanced stage or relapsed disease $(1,5,6,8-10,23)$, but is still associated with significant complication rates. Therefore, recent studies came to underline the fact that pancreatic surgery should be part of the armamentarium of oncological surgeons and gynecologists who deal with ovarian cancer patients. However these procedures should be centralized in specialized, tertiary centers in order to minimize the risk of perioperative complications. However, even in such conditions, pancreatic surgery remains one of the left upper abdominal resections which are associated with the highest risks of perioperative complications, pancreatic leaks and postoperative bleeding being the most common and fearful ones (23). In this respect, we would like to underline once again the fact that the only death which was encountered in our case series was induced by the development of a pancreatic leak after distal pancreatectomy as part of tertiary cytoreduction; in the meantime pancreatic related complications (represented by postoperative hemorrhage after distal pancreatectomy) was also responsive for the need of reoperation of another case submitted to pancreatic resection as part of secondary cytoreduction.

\section{CONCLUSIONS}

Left upper abdominal resections might be needed in order to maximize the debulking effort in the setting of advanced stage and relapsed ovarian cancer. As expected, the proportion of patients who need more extensive surgical procedures in order to achieve RO resection increase with the number of attempts of debulking surgery while the feasibility of complete debulking decreases as the number of attempts increases. In the meantime the risk of developing postopera- 
tive complications as well as their severity seems to increase with the number of surgical attempts, pancreatic and diaphragmatic procedures being most often related to the risk of developing such complications. However, it seems that association of such extended left upper abdominal resections is perfectly justified in order to increase the radicality of the cytoreductive effort.

\section{Acknowledgements}

This work was supported by the project entitled "Multidisciplinary Consortium for Supporting the Research Skills in Diagnosing, Treating and Identifying Predictive Factors of Malignant Gynecologic Disorders", project number PN-III-P1-1.2-PCCDI2017-0833.

In this article, all authors have equal contribution.

\section{REFERENCES}

1. Bacalbasa N, Balescu I, Dima S, Brasoveanu V, Popescu I. Pancreatic Resection as Part of Cytoreductive Surgery in Advanced-stage and Recurrent Epithelial Ovarian Cancer - A Single-center Experience. Anticancer Res. 2015; 35:4125-4129.

2. Bacalbasa N, Balescu I, Dima S, Brasoveanu V, Popescu I. Hematogenous Splenic Metastases as an Independent Negative Prognosis Factor at the Moment of Primary Cytoreduction in Advanced Stage Epithelial Ovarian Cancer - A Single Center Experience. Anticancer Res. 2015; 35:5649-5654.

3. Hoffman MS, Tebes SJ, Sayer RA, Lockhart J. Extended cytoreduction of intraabdominal metastatic ovarian cancer in the left upper quadrant utilizing en bloc resection. Am.J.Obstet.Gynecol. 2007; 197:209-4.

4. Bacalbasa N, Balescu I, Dima S, Brasoveanu V, Popescu I. Splenectomy as Part of Cytoreductive Surgery in Recurrent Epithelial Ovarian Cancer. Anticancer Res. 2015; 35:5097-5101.

5. Kehoe SM, Eisenhauer EL, Abu-Rustum NR, Sonoda Y, D'Angelica M, Jarnagin W R, Barakat RR, Chi DS. Incidence and management of pancreatic leaks after splenectomy with distal pancreatectomy performed during primary cytoreductive surgery for advanced ovarian, peritoneal and fallopian tube cancer. Gynecol.Oncol. 2009; 112:496-500.

6. Bacalbasa N, Balescu I, Vilcu M, Brasoveanu V, Tomescu D, Dima S, Suciu I, Suciu N, Bodog A, Brezean I. Distal pancreatectomy en bloc with splenectomy as part of tertiary cytoreduction for relapsed ovarian cancer. Proceedings of the 4th congress of the romanian society for minimal invasive surgery in ginecology I annual days of the national institute for mother and child health AlessandrescuRusescu, 2019; ISI Proceedings Volume:29-32.

7. Rodriguez N, Miller A, Richard SD, Rungruang B, Hamilton CA, Bookman MA, Maxwell GL, Horowitz NS, Krivak TC. Upper abdominal procedures in advanced stage ovarian or primary peritoneal carcinoma patients with minimal or no gross residual disease: an analysis of Gynecologic Oncology Group (GOG) 182. Gynecol.Oncol. 2013; 130:487-492.
8. Bacalbasa N, Dima S, Balescu I, David L, Brasoveanu V, Popescu I. Results of Primary Cytoreductive Surgery in Advanced-stage Epithelial Ovarian Cancer: A Single-center Experience. Anticancer Res. 2015; 35:4099-4104.

9. Bacalbasa N, Balescu I, Dima S, Herlea V, David L, Brasoveanu V, Popescu I. Initial incomplete surgery modifies prognosis in advanced ovarian cancer regardless of subsequent management. Anticancer Res. 2015; 35:2315-2320.

10. Bacalbasa N, Balescu I, Dima S, Brasoveanu V, Popescu I. The Role of Quaternary Cytoreduction in Recurrent Epithelial Ovarian Cancer: A Single-center Experience. Anticancer Res. 2015; 35:3519-3523.

11. Benedetti PP, Di Donato V, Fischetti M, Casorelli A, Perniola G, Musella A, Marchetti C, Palaia I, Berloco P, Muzii L. Predictors of postoperative morbidity after cytoreduction for advanced ovarian cancer: Analysis and management of complications in upper abdominal surgery. Gynecol.Oncol. 2015; 137:406-411.

12. https://www.sgo.org/wp-content/ uploads/2012/09/FIGO-Ovarian-CancerStaging_1.10.14.pdf.

13. Clavien PA, Barkun J, de Oliveira ML, Vauthey JN, Dindo D, Schulick RD, de Santibanes E, Pekolj J, Slankamenac K, Bassi C, Graf R, Vonlanthen R, Padbury R, Cameron JL, Makuuchi M. The ClavienDindo classification of surgical complications: five-year experience. Ann. Surg. 2009; 250:187-196.

14. Kuhn W, Florack G, Roder J, Schmalfeldt B, Pache L, Rust M, Ulm K, Spathe K, Janicke F, Siewert JR, Graeff $H$. The influence of upper abdominal surgery on peri-operative morbidity and mortality in patients with advanced ovarian cancer FIGO III and FIGO IV. Int J Gynecol Cancer 1998; 8:56-63.

15. Eisenhauer EL, Abu-Rustum NR, Sonoda Y, Levine DA, Poynor EA, Aghajanian C, Jarnagin WR, DeMatteo RP, D'Angelica MI, Barakat R R, Chi DS. The addition of extensive upper abdominal surgery to achieve optimal cytoreduction improves survival in patients with stages IIIC-IV epithelial ovarian cancer. Gynecol.Oncol. 2006; 103:1083-1090.

16. Eisenkop S M, Friedman R L, Wang H J. Complete cytoreductive surgery is feasible and maximizes survival in patients with advanced epithelial ovarian cancer: A prospective study. Gynecol.Oncol. 1998; 69:103-108.

17. Eisenkop SM, Spirtos NM, Friedman RL, Lin WC, Pisani AL, Perticucci S. Relative influences of tumor volume before surgery and the cytoreductive outcome on survival for patients with advanced ovarian cancer: A prospective study. Gynecol.Oncol. 2003; 90:390-396.

18. Bristow RE, Tomacruz RS, Armstrong DK, Trimble EL, Montz FJ. Survival effect of maximal cytoreductive surgery for advanced ovarian carcinoma during the platinum era: A meta-analysis. J.Clin. Oncol. 2002; 20:1248-1259.

19. Martinez A, Pomel C, Mery E, Querleu D, Gladieff L, Ferron G. Celiac lymph node resection and porta hepatis disease resection in advanced or recurrent epithelial ovarian, fallopian tube, and primary peritoneal cancer. Gynecol.Oncol. 2011; 121:258-263.

20. Chi DS, Zivanovic O, Levinson KL, Kolev V, Huh J, Dottino J, Gardner GJ, Leitao MM Jr., Levine DA, Sonoda Y, Abu-Rustum NR, Brown CL, Barakat RR. The incidence of major complications after the performance of extensive upper abdominal surgical procedures during primary cytoreduction of advanced ovarian, tubal, and peritoneal carcinomas. Gynecol.Oncol. 2010; 119:38-42.

21. Sawyer BT, LaFargue CJ, Bristow RE. Extended left upper quadrant resection during primary cytoreductive surgery for Stage IV ovarian cancer. Gynecol.Oncol. 2016; 142:378.

22. Fanfani F, Fagotti A, Gallotta V, Ercoli A, Pacelli F, Costantini B, Vizzielli G, Margariti PA, Garganese G, Scambia G. Upper abdominal surgery in advanced and recurrent ovarian cancer: Role of diaphragmatic surgery. Gynecol.Oncol. 2010; 116:497-501.

23. Di Donato V, Bardhi E, Tramontano L, Capomacchia FM, Palaia I, Perniola G, Plotti F, Angioli R, Giancotti A, Muzii L, Panici PB. Management of morbidity associated with pancreatic resection during cytoreductive surgery for epithelial ovarian cancer: A systematic review. Eur.J.Surg. Oncol. 2019. 